\title{
Le rhapsode et l'ethnographe : Claude Simon en traduction allemande
}

Wolfram Nitsch

\section{OpenEdition}

1 Journals

Édition électronique

URL : http://journals.openedition.org/ccs/966

DOI : $10.4000 /$ ccs.966

ISSN : 2558-782X

Éditeur :

Presses universitaires de Rennes, Association des lecteurs de Claude Simon

\section{Édition imprimée}

Date de publication : 30 avril 2015

Pagination : 183-197

ISBN : 9782753539990

ISSN : $1774-9425$

\section{Référence électronique}

Wolfram Nitsch, "Le rhapsode et l'ethnographe : Claude Simon en traduction allemande », Cahiers Claude Simon [En ligne], 10 | 2015, mis en ligne le 22 septembre 2017, consulté le 24 avril 2019. URL: http://journals.openedition.org/ccs/966; DOI : 10.4000/ccs.966 


\title{
LE RHAPSODE ET L'ETHNOGRAPHE CLAUDE SIMON EN TRADUCTION ALLEMANDE
}

\author{
Wolfram NITSCH \\ Université de Cologne
}

Aux prises avec d'insolites propositions, le traducteur simpatiente. Je le compare à l'écuyer qui prétend faire exécuter à son cheval des mouvements qui ne sont pas naturels à celui-ci. André Gide

\section{D'UN TRADUCTEUR À L'AUTRE}

Si une maison d'outre-Rhin s'aventurait à publier une édition allemande des Euvres de Claude Simon, elle trouverait le terrain bien préparé. Car à l'exception de "La fiction mot à mot ", le seul essai majeur qui reste inaccessible aux lecteurs germanophones de Simon, tous les textes réunis dans les deux volumes de la Pléiade ont été traduits en allemand, certains d'entre eux même plus d'une fois ${ }^{1}$. Voici le bilan remarquable d'une longue histoire sinueuse qui a commencé en 1959, un an avant la première consécration du romancier par un prix littéraire, et qui est parvenue à son terme en 2013, l’année du centenaire de sa naissance. Dans cette période désormais close, l'œuvre romanesque de Simon a beaucoup circulé dans les milieux littéraires allemands, n’occupant

1. Pour une bibliographie détaillée des traductions allemandes de Claude Simon, voir Irene Albers/W. N. (éds.), Lectures allemandes de Claude Simon, Villeneuve d'Ascq, Presses Universitaires du Septentrion, 2013, p. 217-221. Comme dans ce qui suit il n'est question que des romans, il faut signaler aussi la double traduction du Discours de Stockholm dans les deux Allemagnes d'alors: Sinnausdruck Sinnproduktion, traduit par Christian Voigt, Berlin, Akademie der Künste, coll. " Anmerkungen zur Zeit ", 1987; Stockholmer Rede, traduit par Brigitte Burmeister, dans Claude Simon, Anschauungsunterricht, Berlin/Weimar, Aufbau, 1988, p. 133-156. 
pas moins de huit éditeurs et de dix traducteurs différents. On peut néanmoins la diviser en trois étapes.

De 1959 à 1974, la plupart des romans simoniens publiés chez Minuit a été suivie d'assez près d'une version allemande. Cette rapidité plus ou moins prononcée s'explique par un intérêt considérable pour le "nouveau roman " sous l'enseigne duquel la critique rangeait alors ces ouvrages. Ainsi Piper, le premier éditeur allemand de Simon, a publié la traduction du Vent (Der Wind) deux ans après l'original et celle de La Route des Flandres (Die Straße in Flandern) avec un décalage d'un an seulement, tandis que celle du Palace (Der Palast) a tardé jusqu'en 1966. Piper aurait peut-être pu la sortir dans de plus brefs délais s'il l'avait confiée à la traductrice du Vent, Eva Rechel-Mertens, qui avait réussi à

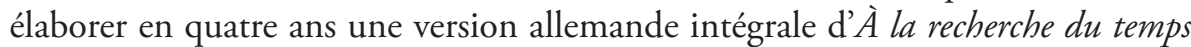
perdu. Mais comme cette élève d'Ernst Robert Curtius était visiblement moins à l'aise avec Simon qu'avec Proust, elle avait été remplacée par Elmar Tophoven, le traducteur de Beckett, qui était de plus en plus pris par la collaboration avec cet autre auteur de Minuit, couronné par le prix Nobel en 1969. Après avoir achevé la traduction de la Route et du Palace, Tophoven n'a donc pu publier celle de L'Herbe (Das Gras) qu'en 1970, c'est-à-dire douze ans après la parution de l'original, et il a dû abandonner celle d'Histoire dès le deuxième chapitre. Cependant, l'éditeur Luchterhand qui avait repris les droits de L'Herbe est arrivé à rétablir l'ancien rythme rapide en engageant de nouveaux traducteurs: Helmut Scheffel pour La Bataille de Pharsale (Die Schlacht bei Pharsalos, 1972) et Irma Reblitz pour Les Corps conducteurs (Die Leitkörper, 1974). Le public allemand était donc assez au courant de l'évolution littéraire de Simon, bien que, à cause du retard de L'Herbe et de son ignorance d'Histoire, il ne pouvait guère deviner le cycle familial qui relie la Route et la Bataille à ces deux romans-là. Par contre, il mesurait sans doute mieux que le public français la dimension autobiographique de l'œuvre simonienne, puisque l'éditeur Suhrkamp avait publié en 1964 Das Seil, une traduction tardive de La Corde raide signée par Eva Moldenhauer.

De 1975 à 1985, en revanche, aucun roman de Simon n'a passé le Rhin. En dépit des critiques en général élogieuses, l'activité éditoriale en faveur de son œuvre s'est arrêtée après la version allemande des Corps conducteurs, soit à cause d'un succès commercial modeste, soit en raison du caractère plus expérimental de ses textes des années soixante-dix. Ainsi, il a fallu attendre plus d'une décennie avant que paraissent chez Rowohlt la traduction de Triptyque par Eva Moldenhauer (Triptychon, 1986), celle de Leçon de choses par Christine Stemmermann (Anschauungsunterricht, 1986) et celle des Géorgiques par Doris Butz-Striebel et 
Trésy Lejoly (Georgica, 1992). En raison de ce silence total, les lecteurs allemands ne pouvaient pas consulter le texte de Triptyque quand, en 1976, la télévision sarroise a montré le film Die SACKGasse (L'impasse), tourné d'après un scénario que Simon avait tiré de ce roman ${ }^{2}$. Et ils avaient encore moins de chance quand ils ont couru les librairies dix ans plus tard, sous le coup de la remise du prix Nobel à Simon, parce que à ce moment-là pas un seul de ses romans n'était disponible.

C'est seulement après le Nobel que l'on est retourné à une publication plus ou moins contemporaine des romans simoniens en langue allemande. La traduction de L'Invitation (Die Einladung, 1988) par Christine Stemmermann ainsi que celles de L'Acacia (Die Akazie, 1991), du Jardin des Plantes (1998) et du Tramway (Die Trambahn, 2002) par Eva Moldenhauer ont paru avec un décalage négligeable par rapport à l'original, de sorte que les lecteurs d'outre-Rhin ont connu les grands romans autobiographiques dans un ordre anachronique, c'està-dire L'Acacia avant Les Géorgiques et Le Jardin des Plantes avant Histoire dont la version allemande, également rédigée par Eva Moldenhauer, a paru avec vingtdeux ans de retard (Geschichte, 1999). Cette nouvelle fidélité à l'œuvre de Simon a été récompensée par des distinctions prestigieuses. Ainsi, Die Akazie autant que Jardin des Plantes ont été rangés en tête de la "SWF-Bestenliste", le palmarès international le plus important de la critique allemande. Encouragé par cette résonance notable, l'éditeur DuMont qui avait succédé à Rowohlt (Die Einladung) et à Suhrkamp (Die Akazie) pour les deux derniers romans de Simon a même commandé une nouvelle traduction de ses quatre premiers romans de Minuit. Réveillée par l'Académie suédoise, l'édition allemande a donc largement compensé la somnolence qu'elle avait montré avant 1985 par rapport à Claude Simon en concédant deux privilèges considérables à ses lecteurs germanophones. D'une part, ils disposent aujourd'hui - comme les lecteurs suédois - d'une traduction homogène d'une grande partie de l'œuvre. Comme la re-traduction du Vent (2001), de L'Herbe (2005), de La Route des Flandres (2003) et du Palace (2006) a été confiée à Eva Moldenhauer qui avait déjà traduit six autres romans, comme c'est encore elle qui a traduit La Chevelure de Bérénice (Das Haar der Berenike, 2006) pour DuMont, Orion aveugle (Blinder Orion, 2008) pour Zweitausendeins et Archipel/Nord (2013) pour Matthes \& Seitz, une seule main a transporté au cours d'un demi-siècle treize œuvres simoniennes dans la langue de Kafka. D'autre part, on peut choisir désormais entre deux versions allemandes de certains ro-

2. À propos de ce film, voir toujours Irene Albers, « Métaphores textuelles et filmiques. Triptyque et L'impasse (Die Sackgasse)", dans I. A./W. N. (éds.), Transports. Les métaphores de Claude Simon, Berne etc., Lang, 2006, p. 305-327. 
mans, voire même entre trois dans le cas de La Chevelure de Bérénice qui avait déjà été traduite en 1985 par Ilma Rakusa et encore une fois en 1994, pour la maison autrichienne Residenz, par Sylvia Ronelt. Peu d'auteurs français du xx siècle, dont Proust et Céline, partagent avec Simon le droit à une polyphonie pareille dans la bibliographie allemande.

\section{DEUX VOIX MAJEURES}

Grâce à la re-traduction de L'Herbe, de La Route des Flandres et du Palace on peut maintenant mieux caractériser en les comparant l'une à l'autre deux voix majeures dans le concert des traducteurs allemands de Simon, Eva Moldenhauer et Elmar Tophoven. Dans leurs versions respectives des trois romans, ces représentants renommés d'un métier longtemps sous-estimé ont mis en relief deux aspects différents de l'écriture simonienne, également importants mais difficiles à respecter dans la même mesure. Ceci se dessine déjà dans les éclaircissements qu'ils ont donnés sur leur travail, assez brefs chez l'auteur de la seconde traduction, très détaillés chez son prédécesseur dont quelques essais ont été réunis dans les mémoires de son épouse Erika Tophoven ${ }^{3}$.

Elmar Tophoven (1923-1989), surnommé " Top " par ses collègues, s'est toujours concentré sur la traduction littéraire. Résidant à Paris depuis 1949, successeur de son ami Paul Celan au poste de lecteur d'allemand à l'École Normale Supérieure depuis 1970, il est devenu « l'avocat du nouveau roman et l'interprète de Samuel Beckett en Allemagne ", comme le résumait l'Académie de Darmstadt en lui décernant le prix de la traduction en 1972. En collaboration avec sa femme, il n'a pas seulement traduit la plupart des œuvres écrites en français de Beckett, de Godot à Mal vu mal dit, mais également de nombreux romans d'Alain Robbe-Grillet et de Nathalie Sarraute. Sa lecture de Claude Simon se situe dans ce contexte. Marquée par la théorie du nouveau roman et par le structuralisme linguistique, elle met en relief la dimension poétique de la prose simonienne, tout en soulignant son affinité avec l'oralité. Pour Tophoven, l'auteur de La Route des Flandres est " un rhapsode dont on peut encore suivre les phrases-méandres avec l'oreille quand l'œil s'est déjà fatigué ${ }^{4}$ ". En conséquence, ses commentaires relatifs à la traduction de Simon portent avant tout sur les structures sémantiques, syntaxiques et phonologiques des textes originaux. D'une part, Tophoven cherche

3. Erika Tophoven, Glückliche Jahre. Übersetzerleben in Paris, Berlin, Matthes \& Seitz, 2011. Cf. aussi son article " Durchläufe », $D u, \mathrm{n}^{\circ}$ 691, 1999, p. 79-80.

4. Ibid., p. 74, traduit par W. N. 
à rendre les nombreuses équivalences sonores qui les parsèment. Comme cela exige une analyse préalable de la prosodie simonienne, il a développé un système de marquage en couleur pour le texte source et le texte cible, inspiré apparemment par le plan de montage pour La Route des Flandres: tandis que le rouge indique des problèmes de vocabulaire et le bleu des difficultés de syntaxe, le vert signale des rimes cachées dans la prose pour lesquelles il faut trouver un équivalent allemand. Une telle rime - virtuelle en l'occurrence - apparaît par exemple au début d'Histoire où il est question de « ténébreux bijoux aux ténébreux éclats dont le nom (jais) évoquait phonétiquement celui d'un oiseau " ( $E$ II, 148). Vu que l'homophonie entre jais et geai est impossible à traduire littéralement, Tophoven l'a compensée - sur le conseil de son collègue Celan - en plaçant avant " Jett ", le mot allemand pour jais, l'adjectif composé " kohlrabenschwarz " (" noir comme un corbeau ») qui à la fois évoque l'oiseau et rétablit par le double /a/ la consonance perdue. Tout ceci ressort d'une fiche appartenant à l'immense fichier que Tophoven a créé à partir de 1970 pour détecter les structures de l'original et pour documenter les solutions du traducteur ${ }^{5}$. Afin de rendre transparent le travail de celui-ci, de rendre accessibles à tout le monde les arcanes du métier en les mettant "sous verre", il s'est même initié dix ans plus tard au traitement de texte dont les milieux littéraires d'alors ignoraient encore les ressources. En outre, il avait déjà commencé vers 1960 à enregistrer ses traductions sur magnétophone pour éprouver leur qualité sonore. Comme le Krapp de Beckett et peut-être inspiré par son exemple, "Top » parlait sans cesse sur bande, mais toujours en lisant un texte préparé qu'il passait pour ainsi dire au gueuloir électrique. À l'entendre, le résultat persuadait même des lecteurs que le texte écrit avait laissés perplexes, sans doute aussi grâce à la voix bien formée du traducteur qui à ses débuts avait doublé des films documentaires. Dans les documents sonores qu'il a légués, Tophoven arrive en effet à faire valoir la qualité « rhapsodique » de la prose simonienne - bien plus d'ailleurs que l'auteur lui-même qui n'aimait guère lire à haute voix, mais qui a accepté quand même de faire une lecture publique bilingue du Palace avec son traducteur en 1968, enregistrée par la télévision sarroise ${ }^{6}$. Une autre raison pour laquelle la version allemande séduit davantage l'auditeur réside peut-être dans la syntaxe. Car tout en essayant de rendre les nombreuses consonances de la prose

\footnotetext{
5. Voir Elmar Tophoven, "Quand le monde dépend des points et des virgules " (1985), traduit par Laurent Cassagnau et présenté par Irene Albers, dans Lectures allemandes de Claude Simon, op. cit., p. 55-60, ici p. 59.

6. " Deutsch-französische Begegnungen in Saarbrücken: Claude Simon und Elmar Tophoven ", Saarländischer Rundfunk, 18 avril 1968, 3'53". La réticence de Simon par rapport au " gueuloir » de Flaubert, qui à son avis donne trop d'emphase au style, ressort de son entretien avec Aliette Armel dans Le Magazine littéraire, $\mathrm{n}^{\circ} 401$, 2001, p. 57: «concevrait-on Proust "gueulant" la Recherche?».
} 
simonienne, Tophoven s'efforce d'autre part de réduire sensiblement le nombre des participes présents qui, à son avis, détonnent dans une phrase allemande. En 1985, il a certes déclaré qu'il fallait les conserver "le plus possible " parce qu'ils avaient pour fonction "de renforcer l'effet immédiat de ce qui est ressaisi et de faire participer davantage le lecteur à l'action ${ }^{7}$ ". Mais, comme il l'avoue dans ses essais antérieurs, sa pratique générale consiste à remplacer les participes présents par d'autres structures syntaxiques plus courantes dans sa langue maternelle; il cite en exemple un paragraphe de sa traduction de La Route des Flandres où il n'y a plus que deux participes présents par rapport à deux douzaines dans l'original ${ }^{8}$. En cas de doute, il préfère envoûter le lecteur allemand au lieu de le dépayser outre mesure.

À la différence d'Elmar Tophoven, Eva Moldenhauer ne s'est jamais spécialisée dans les textes littéraires. Enracinée dans le milieu intellectuel de Francfort et longtemps proche de la maison Suhrkamp où elle a dirigé avec son mari Karl Markus Michel une importante édition des œuvres de Hegel, elle a traduit du français autant d'ouvrages de théorie que de fiction. Si elle a fait découvrir aux lecteurs allemands des romanciers comme Jorge Semprún, Rachid Boudjedra ou Pierre Michon, elle a fait de même pour des anthropologues comme Marcel Mauss, Claude Lévi-Strauss ou Philippe Descola. C'est pour la traduction d'un ouvrage de ce dernier, Par-delà nature et culture, qu'elle a reçu en 2011 le prix Raymond Aron. Mais il est révélateur, que dans le discours de réception prononcé à cette occasion, elle revienne à ses traductions de Claude Simon qui lui avaient déjà valu d'autres distinctions, par exemple le prix Celan pour Die Akazie ou le prix de la foire de Leipzig pour Das Gras. Car elle y présente Simon comme un ethnographe du monde moderne qui loin de dénigrer le référent matériel - à la façon des porte-parole du nouveau roman parodiés dans Le Jardin des Plantes (CE I, 1163) - le met au contraire en valeur dans ses descriptions minutieuses de certains lieux et de certains spectacles ${ }^{9}$. Par conséquent, le traducteur est obligé de repérer des traces extra-littéraires. Dans son discours, Moldenhauer raconte par exemple comment elle a fait des recherches sur place en traduisant Le Vent et Le Tramway. À l'instar du protagoniste de La Bataille de Pharsale qui essaie de trouver le champ de bataille décrit chez César et Lucain, elle est allée à Perpignan pour y identifier une place évoquée dans le premier roman avec l'aide d'un

7. "Quand le monde dépend des points et des virgules ", art. cit., p. 57.

8. Cf. Glückliche Jahre, op. cit., p. 76-77, 171.

9. Voir Eva Moldenhauer, "Aus der Übersetzerwerkstatt", dans Raymond-Aron-Preis für deutsch-französische Übersetzungen aus den Geistes- und Sozialwissenschaften, Stuttgart, DVA-Stiftung, 2011, p. 43-47. 
témoin appartenant à la génération de Simon, ou pour reconstruire le trajet du tramway évoqué dans le dernier roman en consultant un album de cartes postales historiques qu'elle avait trouvé, à la grande joie du romancier lui-même, dans l'office de tourisme de Canet-Plage. En outre, la traductrice rend compte de ses enquêtes sur certains termes techniques employés dans Le Tramway et La Route des Flandres. Pour rendre exactement les descriptions du tramway dans le roman du même titre, elle a visité un musée consacré à ce véhicule moderne et consulté un journaliste spécialisé dans l'histoire des transports; et pour mieux saisir les descriptions de cavaliers militaires et civils qui abondent dans l'autre roman, elle a assisté à une course de chevaux et interrogé une ancienne femme jockey. Si l'atelier d'Elmar Tophoven ressemble à un laboratoire linguistique, celui d'Eva Moldenhauer rappelle donc plutôt un centre de documentation sur l'histoire des mœurs du $\mathrm{xx}^{\mathrm{e}}$ siècle.

\section{TRADUCTION ET RE-TRADUCTION}

Les préoccupations différentes que trahissent les commentaires des deux traducteurs sur leur travail se manifestent également dans les textes auxquels il a abouti, notamment dans les deux versions allemandes de La Route des Flandres. Ceci peut être étudié dans un passage cité par Tophoven pour souligner les vertus "rhapsodiques» de la prose simonienne. Il apparait dans la seconde partie du roman où le narrateur évoque la course d'obstacles que son ancien capitaine de Reixach avait risquée avant la guerre, à la place de son jockey Iglésia et devant les yeux de sa jeune femme Corinne qui l'avait peut-être trompé avec celui-ci. Quoiqu'il s'agisse d'une pure imagination rétrospective du narrateur hanté par le débâcle militaire sur la route des Flandres, la compétition sportive est décrite en détail : d'abord la lente cavalcade des jockeys en direction du starter, et ensuite, après une brusque irruption du souvenir de l'embuscade allemande, leur course rapide sur le circuit semé de haies. C'est dans cette suite de la description que se situe le passage en question qui évoque l'apparition du peloton derrière les arbres du turf et son mouvement vers la tribune:

Ils apparurent enfin après le dernier arbre, toujours dans le même ordre, la tache, la pastille rose toujours en même position tandis qu'ils entamaient la dernière partie du tournant, le peloton se muant peu à peu en une masse confuse (les derniers semblant rattraper les premiers) qui, tout au fond de la ligne droite, ne fut plus qu'une houle, un moutonnement de têtes montant et descendant sur place, les chevaux agglomérés en paquet paraissant un moment ne plus avancer (simplement les toques des jockeys montant et descendant) jusqu'à ce que soudain le premier cheval non pas franchît mais crevât la haie, c'est-à-dire que brusquement il fut là, les deux 
pattes de devant projetées devant lui, raides, jointes ou plutôt l'une d'elles légèrement en avant de l'autre, les deux sabots pas tout à fait à la même hauteur, le cheval engagé jusqu'à mi-corps entre les fagots bruns qui surmontaient la barrière, reposant apparemment sur le ventre comme en équilibre, une fraction de seconde immobile, aurait-on dit, jusqu'à ce qu'il basculât en avant tandis qu'un second, puis un troisième, puis plusieurs ensemble, tous figés successivement en équilibre, dans cette position de cheval à bascule, apparaissent, s'immobilisent, s'inclinent en avant, retrouvant le mouvement en même temps que le contact avec la terre, le peloton galopant maintenant, de nouveau soudé, vers les tribunes, grossissant, franchissant l'obstacle suivant, puis ce fut là: l'espèce de tonnerre silencieux, la sourde trépidation du sol sous les sabots, les mottes de gazon, volant loin derrière, les soyeuses casaques froissées claquant dans le vent de la course et les bustes des jockeys penchés sur l'encolure, non pas immobiles comme ils paraissaient dans la ligne opposée, mais oscillant légèrement d'avant en arrière au rythme des foulées, avec leurs identiques bouches ouvertes cherchant l'air, leur identique aspect de poissons hors de l'eau, à demi asphyxiés, passant devant les tribunes entourés ou plutôt enveloppés par cette attentive chape de vertigineux silence qui semblait les isoler (les quelques cris fusant de la foule paraissant - et non pas aux oreilles des jockeys mais à celles des spectateurs eux-mêmes - parvenir de très loin, futiles, vains, incongrus et aussi faibles que des bégaiements inarticulés de petits enfants), les accompagner, laissant derrière eux, bien après leur passage, comme un persistant sillage de silence à l'intérieur duquel le martèlement des sabots allait diminuant, s’amenuisant, seulement crevé, sporadiquement, par le claquement sec (comme le bruit d'une branche cassée) d'un coup de cravache, de minuscules détonations s'éloignant elles aussi, décroissant, le dernier cheval franchissant la haie vive couronnant la légère montée, exactement comme un lapin, l'image de son arrière-train en position de ruade restant un moment sur la rétine, immobilisée, et disparaissant enfin [...] (E I, 313-314).

\section{Voici la traduction du passage par Elmar Tophoven, paru en 1961 et cité par lui-même dans un essai sur le nouveau roman qu'il a écrit quelques années plus tard :}

Sie erschienen nun hinter dem letzten Baum, immer noch in der gleichen Reihenfolge, der Fleck, die rosa Pastille immer noch an vierter Stelle, während sie den letzten Teil der Biegung zurücklegten, dann verwandelte sich der Haufe allmählich in eine undeutliche Masse (die letzten schienen die ersten einzuholen), die ganz am Anfang der geraden Strecke nur noch eine einzige Welle, ein Gewoge von auf der Stelle steigenden und sinkenden Köpfen war, wobei die zu einem Packen verschmolzenen Pferde einen Moment nicht vom Fleck zu kommen schienen (da nur die Jockeikappen stiegen und sanken) bis das erste Pferd blitzschnell die Hecke nicht übersprang sondern durchsprengte, da es plötzlich da war, mit vorgestreckten, steifen Vorderbeinen, beide nebeneinander oder vielmehr eins ein wenig vor dem anderen und beide Hufe nicht ganz in gleicher Höhe, während das Pferd bis zur Hälfte seines Leibes zwischen den die Schranke überragenden braunen Reisern steckte und scheinbar wie im Gleichgewicht auf dem Bauch ruhte, für den Bruchteil einer Sekunde regungslos, wie man hätte meinen können, bis es nach vorn kippte indes ein zweites, dann ein drittes, dann mehrere zugleich, alle nacheinander wie im Gleichgewicht, in jener Schaukelpferdposition erstarrt erschienen, verharrten, vornüberkippten und bei Berührung des Bodens wieder in Bewegung gerieten, der Haufe galoppierte nun, erneut zusammengeschweißt, auf die Tribünen zu, größer werdend, das nächste Hindernis nehmend, dann war es so weit: eine Art gedämpfter Donner, das dumpfe Dröhnen des Bodens unter den Hufen, die weit zurückfliegenden Rasenklumpen, die im Rennwind 
flatternden knitterigen seidenen Jacken und die Rümpfe der über die Hälse gebeugten Jockeis, nicht regungslos wie sie auf der gegenüberliegenden Bahn aussahen, sondern im Rhythmus der Galoppsprünge leicht von vorn nach hinten schwindend, mit ihren gleichförmig aufgesperrten nach Luft schnappenden Mündern, ihrem gleichförmigen Aussehen aus dem Wasser gezogener Fische, halb erstickt, an den Tribünen vorbeischießend, umgeben oder vielmehr umhüllt von jenem Mantel gespannter schwindelerregender Stille die sie gleichsam isolierte (die wenigen Rufe die aus der Menge aufstiegen schienen - und zwar nicht in den Ohren der Jockeis sondern in denen der Zuschauer - von weither zu kommen und überflüssig, unnütz, unpassend und so leise wie unartikuliertes Gelispel kleiner Kinder zu sein), sie gleichsam begleitete und lange nach ihrem Vorbeischnellen noch eine Art Stille hinter sich herschleppte in der das Hämmern der Hufe abnahm, verklang und nur noch hin und wieder von dem kurzen, klatschenden Geräusch (wie dem eines knackenden Zweigs) eines Reitpeitschenschlags, von kaum hörbarem Knallen unterbrochen wurde, das sich ebenfalls verhallend entfernte, während das letzte Pferd die lebende Hecke oben auf der leichten Steigung wie ein Hase übersprang wobei das Bild seiner ausschlagenden Hinterhand einen Moment immobilisiert auf der Netzhaut haftete, und endlich verschwand ${ }^{10}[\ldots]$.

\section{Dans la re-traduction du roman par Eva Moldenhauer qui a paru en 2003, le même passage se lit ainsi :}

Sie erschienen schließlich hinter dem letzten Baum, immer noch in derselben Reihenfolge, der Fleck, die rosa Pastille immer noch an vierter Stelle während sie den letzten Teil der Biegung in Angriff nahmen, das Feld sich allmählich in eine undeutliche Masse verwandelte (die letzten schienen die ersten einzuholen) die ganz hinten auf der geraden Bahn nur noch eine Dünung war, ein Gewoge sich auf der Stelle hebender und senkender Köpfe, wobei die zu einem Pakken zusammengeklumpten Pferde einen Moment lang nicht mehr voranzukommen schienen (lediglich die Kappen der Jockeis hoben und senkten sich) bis plötzlich das erste Pferd die Hekke nicht überwand sondern durchbrach, das heißt daß es plötzlich da war, die beiden Vorderbeine vorgestreckt, steif, nebeneinander oder vielmehr eins leicht vor dem andern, die beiden Hufe nicht ganz auf gleicher Höhe, das Pferd bis zur Hälfte des Körpers zwischen den auf der Hürde angebrachten braunen Reisern scheinbar wie im Gleichgewicht auf dem Bauch ruhend, für den Bruchteil einer Sekunde regungslos, so hätte man meinen können, bis es nach vorn kippte während ein zweites, ein drittes, mehrere zugleich, alle nacheinander wie im Gleichgewicht in jener Schaukelpferdposition erstarrt, auftauchten, verharrten, sich vornüberbeugten, bei der Berührung des Bodens wieder in Bewegung gerieten, das Feld jetzt galoppierte, erneut zusammengeschweißt, auf die Tribünen zu, größer werdend, das nächste Hindernis nehmend, dann war es da: eine Art lautloses Donnern, das dumpfe Beben des Bodens unter den Hufen, die weit zurückfliegenden Rasenklumpen, die im Rennwind flatternden zerknitterten seidenen Blusen und die über die Pferdehälse gebeugten Oberkörper der Jockeis, nicht reglos wie sie auf der gegenüberliegenden Bahn wirkten, sondern im Rhythmus des Galopps leicht vor und zurück schwankend mit ihren alle nach nach Luft schnappenden offenen Mündern, alle mit dem Aussehen von Fischen außerhalb des Wasser, halb erstickt, an den Tribünen vorbeipreschend, umgeben oder vielmehr umhüllt von jenem Mantel schwindelerregender Stille der sie gleichsam isolierte (denn die wenigen aus der Menge aufsteigenden Schreie schienen - nicht in den

10. Die Straße in Flandern, Munich, Piper, 1985, p. 166-167. Cf. Elmar Tophoven, "Zeitgenössische französische Prosa in der Sicht eines Übersetzers " (1967), dans Glückliche Jahre, op. cit., p. 151-181, ici p. 173-174. 
Ohren der Jockeis sondern in denen der Zuschauer selbst - von weither zu kommen, belanglos, vergeblich, unpassend und so leise wie das unartikulierte Lallen kleiner Kinder), sie gleichsam begleitend und hinter ihnen, noch lange nach ihrem Vorbeiritt, eine Art Stille zurücklassend in der das Hämmern der Hufe abnahm, verebbte, sporadisch noch durchbrochen von dem kurzen Knallen (gleich dem Geräusch eines zerbrechenden Asts) eines Peitschenschlags, winzige Detonationen die sich ebenfalls entfernten, sich abschwächend, indes das letzte Pferd die Hecke oben auf der leichten Steigung übersprang, genau wie ein Kaninchen, das Bild seiner ausschlagenden Hinterhand einen Augenblick reglos auf der Netzhaut haften blieb und schließlich verschwand ${ }^{11}[\ldots]$.

En comparant les deux versions, on note d'abord que Moldenhauer reprend littéralement quelques solutions de son prédécesseur à qui elle rend d'ailleurs hommage dans un bref remerciement qui fait office de postface. Comme Tophoven, elle traduit le mot "moutonnement" qui apparaît au début par "Gewoge ", une expression plutôt rare en allemand; et par la suite, elle copie même un syntagme entier de la version antérieure, rendant "vers les tribunes, grossissant, franchissant l'obstacle suivant " de son côté par "auf die Tribünen zu, größer werdend, das nächste Hindernis nehmend ». Au lecteur qui consulte la première traduction, elle signale donc qu'elle considère la sienne comme doublement seconde, par rapport à l'original et par rapport à une version allemande de l'époque. Mais c'est justement cette référence double qui selon le traductologue Antoine Berman permet à la re-traduction d'être plus littérale et à cet égard plus fidèle à l'original que la traduction précédente ${ }^{12}$. En effet, Moldenhauer fait preuve d'une plus grande précision lexicale dans le passage en question, bien qu'elle ne connaisse que de seconde main le monde des cavaliers - contrairement à Tophoven qui partageait avec Simon l'expérience de la cavalerie militaire. Tandis que celui-ci traduit "peloton" par "Haufe ", une expression assez générale et un peu désuète, elle choisit le terme "Feld " qui appartient au vocabulaire du journalisme sportif et s'intègre mieux à la description de la course. Et là où Simon compare les cris des jockeys à de faibles « bégaiements inarticulés de petits enfants", elle opte pour le mot "Lallen" qui correspond davantage à l'idée d'un balbutiement de bébé que le mot "Gelispel " (" chuchotement ») employé par Tophoven. Une préférence semblable pour les solutions littérales caractérise la syntaxe de la re-traduction. Peut-être encouragée par la critique qui avait salué Helmut Scheffel pour sa fidélité au participe présent simonien ${ }^{13}$, Eva

11. Die Straße in Flandern, Cologne, DuMont, 2003, p. 182-184.

12. Cf. Antoine Berman, La traduction et la lettre ou l'auberge du lointain (1985), Paris, Le Seuil, coll. «L'ordre philosophique ", 1999, p. 104-105.

13. Cf. Gerda Zeltner, "Im Zeichen der Akazie. Welt und Sprache Claude Simons », Neue Zürcher Zeitung, 14 juin 1991, p. 43. 
Moldenhauer conserve plus souvent que le premier traducteur de La Route des Flandres cette construction peu usitée en allemand. À quatre endroits du passage en question, elle renonce à la transformer comme Tophoven en une proposition basée sur un verbe au mode personnel. Comme l'exemple le plus frappant pour cette tendance, on peut citer la description simonienne de la " chape de vertigineux silence " qui semble "isoler " les jockeys des spectateurs, les "accompagner" et faire d'eux pour ainsi dire des émetteurs d'anti-bruit «laissant derrière eux, bien après leur passage, comme un persistant sillage de silence ». La traduction de 1961 rend ce dernier syntagme par une proposition relative dépendant de "silence " et par là quelque peu tautologique: "Stille die [...] sie gleichsam begleitete und lange nach ihrem Vorbeischnellen noch eine Art Stille hinter sich herschleppte ". Celle de 2003 garde au contraire le participe présent "laissant " et lui en antépose même un autre, absent de l'original, sans doute pour atténuer la tautologie du silence se suivant à lui-même qu'elle ne sait pas non plus éviter: "sie gleichsam begleitend und hinter ihnen, noch lange nach ihrem Vorbeiritt, eine Art Stille zurücklassend" (mes italiques). Avec des solutions pareilles, Eva Moldenhauer se range du côté du camp des "sourciers " qui dans la terminologie de Jean-René Ladmiral s'opposent aux "ciblistes » en s'approchant de la lettre de l'original, quitte à déconcerter les lecteurs de la traduction ${ }^{14}$. Tandis que Tophoven tend à réduire l'effet de distanciation propre à la phrase simonienne et même sensible pour un lecteur français, elle le fait ressortir au point d'être parfois plus simonienne que Simon lui-même.

Si la seconde traduction s'éloigne moins du lexique et de la syntaxe de $\mathrm{La}$ Route des Flandres, la première reste en revanche plus fidèle à la sonorité suggestive du texte source. Guidé par l'idée que Simon est un "rhapsode " moderne, Tophoven conserve les nombreuses allitérations et consonances qui parsèment la description de la course de chevaux, soit en les imitant sur place, soit en les compensant ailleurs, suivant une règle déjà établie par Du Bellay ${ }^{15}$. Quand par exemple l'original dit du peloton compact qu'il n'est plus qu' « une houle, un moutonnement de têtes ", sa version parle de " eine einzige Welle, ein Gewoge ", substituant la répétition du /ou/ par celle d'un /w/ ; cet effet se perd dans la

14. Voir maintenant Jean-René Ladmiral, Sourcier ou cibliste, Paris, Les Belles Lettres, 2014. Comme beaucoup d'autres concepts de la traductologie, cette distinction remonte aux réflexions de Friedrich Schleiermacher dans son essai Des différentes méthodes du traduire (1813), traduit par Antoine Berman, Paris, Le Seuil, coll. « Points Essais », 1999.

15. "Ce que le traducteur n'a pu rendre à ses bonnes grâces à un endroit, qu'il s'efforce de le récompenser en l'autre", cité dans Berman, La traduction et la lettre, op. cit., p. 37. À propos de cette règle, voir aussi Umberto Eco, Dire presque la même chose. Expériences de traduction, Paris, Grasset, 2007, chap. 5. 
version ultérieure qui rend "houle " par " Dünung ", un mot certes plus précis mais bien moins évocateur. Plus réussie encore me semble la solution de Tophoven pour l'attribut que Simon assigne à la galopade tonitruante des jockeys, "l'espèce de tonnerre silencieux, la sourde trépidation du sol sous les sabots". En proposant les expressions paronymes " gedämpfter Donner » et « das dumpfe Dröhnen ", il arrive à imiter le piétinement des chevaux dans l'hippodrome au même degré que le romancier fasciné par les effets onomatopoétiques et les mots évocateurs ${ }^{16}$. Avec les mêmes moyens, il met en relief la violence sous-jacente du concours hippique qui dans la mémoire du narrateur se superpose au combat des dragons. Là où l'original dit «jusqu'à ce que soudain le premier cheval non pas franchît mais crevât la haie ", la première traduction met "blitzschnell die Hecke nicht übersprang sondern durchsprengte", rendant crever par le verbe insolite durchsprengen qui non seulement ressemble à überspringen (franchir), mais évoque en même temps sprengen (dynamiter), donc la véhémence de la guerre moderne. Ce que la seconde traduction ne signale qu'à la fin du passage, où elle rend littéralement par «Detonationen » les " détonations " que provoquent les coups de cravache des cavaliers, se profile ici dès le début.

Tout compte fait, il semble difficile de soutenir que l'une des deux versions allemandes de La Route des Flandres soit plus réussie que l'autre. Chacune d'elles repose sur une lecture lucide de la prose simonienne. Si la traduction de Tophoven souligne la sonorité évocatrice de celle-ci, la re-traduction de Moldenhauer détache sa précision descriptive et son étrangeté syntactique; au rhapsode silencieux qui fascine le lecteur succède l'ethnographe littéraire qui le dépayse. Il s'agit donc de deux versions au sens fort du terme sur lequel insiste Borges en caractérisant les différentes traductions d'Homère comme des "perspectives diverses sur un fait mobile ${ }^{17}$. Les deux traductions de la page sur la course de chevaux n'enrichissent pas seulement la langue maternelle de leurs auteurs que l'on pourrait comparer pour cela "à l'écuyer qui prétend faire exécuter à son cheval des mouvements qui ne sont pas naturels à celui-ci ", selon la boutade célèbre d'André Gide ${ }^{18}$. Elles enrichissent en même temps le texte original que le spécialiste a l'habitude de lire sans aucun égard pour ce qu'il devient dans une

16. Sur le "cratylisme secondaire " de Simon qu'il partage avec Michel Leiris, voir mon étude " Une poétique de la dépense. Claude Simon et le Collège de Sociologie ", Cahiers Claude Simon, nº 4, 2008, p. 33-52, en particulier p. 42-46.

17. Jorge Luis Borges, "Las versiones homéricas" (1932), dans Obras completas, éd. Carlos V. Frías, Buenos Aires, Emecé, 1989, t. 1, p. 239-243, ici p. 239: "diversas perspectivas de un hecho móvil ».

18. André Gide, "Avant-propos », dans Shakespeare, Euvres complètes, éd. Henri Fluchère, Paris, Gallimard, "Bibliothèque de la Pléiade ", 1959, t. 1, p. 188-198. 
langue étrangère. C'est sans doute aussi sous ce rapport qu'il faut interpréter l'observation de Claude Simon adressée à sa traductrice allemande la plus constante: «la traduction n'est pas un simple travail de transcription, comme on le croit vulgairement, mais un véritable travail de production $^{19} "$.

19. Lettre de Claude Simon à Eva Moldenhauer du 8 juin 1998, citée avec l'aimable autorisation de la destinatrice. 


\section{BIBLIOGRAPHIE DES ROMANS DE SIMON EN TRADUCTION ALLEMANDE}

1959

Der Wind. Versuch der Wiederherstellung eines Barockaltars (Le Vent), Eva Rechel-Mertens, Munich, Piper.

1961

Die Straße in Flandern (La Route des Flandres), Elmar Tophoven, Munich, Piper.

1964 Das Seil (La Corde raide), Eva Moldenhauer, Francfort-sur-leMain, Suhrkamp.

Der Palast (Le Palace), Elmar Tophoven, Munich, Piper.

1970 Das Gras (L'Herbe), Erika et Elmar Tophoven, Neuwied, Luchterhand.

1972 Die Schlacht bei Pharsalos (La Bataille de Pharsale), Helmut Scheffel, Neuwied, Luchterhand.

1974 Die Leitkörper (Les Corps conducteurs), Irma Reblitz, Neuwied: Luchterhand.

1985 Das Haar der Berenike (La Chevelure de Bérénice), Ilma Rakusa, Akzente, 32, 1985, n 1, p. 9-19.

1986 Triptychon (Triptyque), Eva Moldenhauer, Reinbek, Rowohlt.

1986 Anschauungsunterricht (Leçon de choses), Christine Stemmermann, Reinbek, Rowohlt.

1988 Die Einladung (L'Invitation), Christine Stemmermann, Reinbek, Rowohlt. 
1991 Die Akazie (L'Acacia), Eva Moldenhauer, Francfort-sur-le-Main, Suhrkamp.

1992 Georgica (Les Géorgiques), Doris Butz-Striebel et Trésy Lejoly, Reinbek, Rowohlt.

1994 Das Haar der Berenike (La Chevelure de Bérénice), Sylvia Ronelt, Salzburg, Residenz.

1998 Jardin des Plantes (Le Jardin des Plantes), Eva Moldenhauer, Cologne, DuMont.

1999 Geschichte (Histoire), Eva Moldenhauer, Cologne, DuMont.

2001 Der Wind (Le Vent), Eva Moldenhauer, Cologne, DuMont.

2002 Die Trambahn (Le Tramway), Eva Moldenhauer, Cologne, DuMont.

2003 Die Straße in Flandern (La Route des Flandres), Eva Moldenhauer, Cologne, DuMont.

2005 Das Gras (L'Herbe), Eva Moldenhauer, Cologne, DuMont.

2006 Der Palast (Le Palace), Eva Moldenhauer, Cologne, DuMont.

2006 Das Haar der Berenike (La Chevelure de Bérénice), Eva Moldenhauer, Cologne, DuMont.

2008 Der blinde Orion (Orion aveugle), Eva Moldenhauer, Francfortsur-le-Main, Zweitausendeins.

2013 Archipel/Nord. Kleine Schriften und Photographien, Eva Moldenhauer, Berlin, Matthes \& Seitz. 